\title{
VIBRATION ANALYSIS OF A PIEZOELECTRIC CYLINDER PANEL
}

\author{
BÉLA KovÁCS \\ Institute of Mathematics, University of Miskolc, \\ 3515 Miskolc-Egyetemváros, Hungary \\ matmn@uni-miskolc.hu
}

[Received: August 31, 2006]

\begin{abstract}
A new iterative model is presented for the dynamic analysis of a piezoelectric cylinder panel. The differential equations which govern the free vibrations of a piezoelectric panel as well as the boundary conditions are derived from Hamilton's principle by taking the bending, shear and normal deformation into account. The author developed a new iterative process to successively refine the stress and strain fields and the electrical field in the panel. The model includes the effects of transverse shear and rotary inertia. The iterative model can be applied to predict the modal frequencies and the stress field in the piezoelectric medium under the conditions of cylindrical bending and periodic motion.
\end{abstract}

Mathematical Subject Classification:

Keywords: piezoelectric cylinder panel, stress and strain fields, electrical field, iterative model

\section{INTRODUCTION}

In recent years piezo-eletric materials and their use in structural dynamic have become a growing area of research. These materials induce electric potential / charge when they are subjected to mechanical deformations by virtue of direct piezoeletric effect and are deformed due to the externally applied voltage by virtue of converse piezoeletric effect. The load-bearing structures fitted with piezoelectric layers, called intelligent structures, are subjected to the coupled action of electric and elastic fields.

The theory of piezoelectricity was first formulated by Voigt in 1894 . The basic theory of linear piezoelectricity has been outlined by several authors [1, 2, 3, Early computational studies of piezoelectric solids include those of Eer Nisse 4 and Holland [5] for electroelastic vibration analysis using Ritz method. The work of Tiersten [6] provided the theoretical foundations and numerous examples of the dynamic behavior of piezoelectric plates. Finite element modelling of the electroelastic behaviour of a piezoelectric sensor/actuator structural system was presented by Tzou and Tseng [7] and Tzou el al. 8]. Robbins and Reddy 9] developed the finite element models of a piezoelectrically actuated beam using four different displacement-based onedimensional beam theories, derivable from the generalized laminated plate theory of 
Reddy [10] to investigate the behaviour of a piezoelectrically actuated beam. Very few exact solutions of the three-dimensional field equations are available for the coupled response of piezoelectric elements to electromechanical loading. Ray el al. [10, 12] presented exact solutions for the static analysis of a simply-supported piezoelectric plate and a layered intelligent plate under cylindrical bending. A three-dimensional analysis of semi-infinite crack embedded in a transversely isotropic piezoelectric material was performed by Sosa and Pak [13. Heylinger and Brooks [14] have presented exact solutions for the free vibration behavior of piezoelectric laminates in cylinder bending. The equations of motion, the charge equation, and the boundary and interface conditions are satisfied exactly. A discrete-layer laminate model is presented for the analysis of laminated piezoelectric composite shells in [15]. The variational formulation and corresponding finite element model are developed using the equations of motion and the charge equation. Páczelt and Kovács [16] have studied a semicircular planar prismatic beam subjected to bending moments resulting from piezoelectric actuator devices bonded to the parallel surfaces of the beam. The bending and torsional deformations are investigated with the Finite Element Method and the closed-form solution of the linear theory of 3D curved beams, respectively.

The predictor-corrector procedures appear to have high potential for the accurate prediction of vibration frequencies, stresses and deformations in multilayered composite plates end shells. Zapfe and Lesieutre [17] developed an iterative process to refine successively the shape of the stress/strain distribution for the dynamic analysis of laminated beams. The iterative model is used to predict the modal frequencies and damping of simply supported beams with integral viscoelastic layers. Noor and Burton [18 presented a predictor-corrector approach for the analysis of composite plates. The authors used a plate model based on first-order shear deformation theory, coupled with integration of the equilibrium equations, to refine the estimate of the local stress field through the thicknesss of the laminate. The refined stress field was also used to generate improved estimates of the shear correction factors in the first-order shear deformation model, leading to improved estimates of the plate displacements and natural frequencies. Lee and Cao [19] have presented a predictor-corrector approach for the numerical analysis of general thick laminated plates which ensures that the transverse shear stress distributions from both constitutive and equilibrium considerations are sufficiently close to each other before the computations of the displacements and stresses are considered sufficiently accurate. In the predictor phase, a linear or cubic zig-zag model is adopted and the layerwise polynomial approximation of the transverse shear stresses through the thickness is determined from the equilibrium equations of elasticity.

The present research extends the iterative model developed by Zapfe and Lesieutre to the dynamic analysis of a cylinder panel of piezoelectric medium. The current model is developed for the specific case of a simply supported cylinder panel under the conditions of cylindrical bending and periodic motion. 


\section{Equations of motion}

The geometry of interest and the notations are shown in Figure 1. We consider an infinitely long (perpendicular to the plane of paper) cylinder panel of piezoelectric medium. The free vibration of the cylinder panel is confined to the $r-\varphi$ plane. The $z$ axis is out-of-plane, with the $r$ and $\varphi$ coordinates corresponding to the respective radial and circumferential directions of the panel. Owing to the cylinder panel is assumed to be infinitely long in the $z$-direction, the piezoelectric medium is under cylindrical bending ( i.e. plane strain state ) in the $r-\varphi$ plane. As indicated in the Figure, the panel ends are simply supported as described by the end conditions. The panel is considered to be thin and of radius of curvature $R$ of the middle surface, and thickness $h$. The arc length $s$ is measured along the middle surface of the panel in the circumferential direction, which is defined by $s$ (Fig.1), where $s=R \varphi$. The length of the panel in the circumferential direction is denoted by $L$. In this case $L=R \vartheta$ where $\vartheta$ is the opening angle of the curved panel. The displacements of the panel in the $\varphi-$ and $r$ - directions are denoted by $u$ and $w$ respectively. Under the conditions of cylindrical bending (i.e. plane strain state), the displacements field takes the form $\mathbf{t}=w(r, \varphi, t) \mathbf{e}_{r}+u(r, \varphi, t) \mathbf{e}_{\varphi}$, and the electrostatic potential has the form $\Phi=\Phi(r, \varphi, t)$. Hence all variables are independent of the $z$ coordinate, and terms containing a gradient in the $z$-direction vanish. For the analysis of the response of piezoelectric structural components we need to deal with two different field equations that are coupled to each other. One is the balance of the momentum equation and the other is the balance of the charge equation. These two field equations are coupled to each other through the constitutive equations of the material.

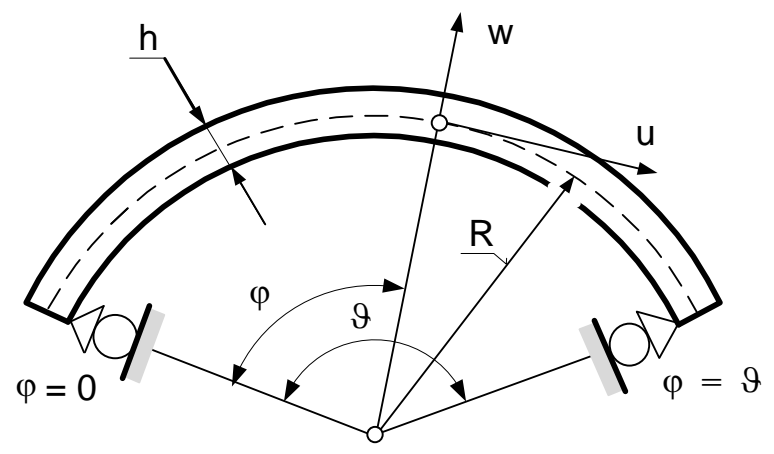

Figure 1. Parameters for a cylinder panel.

We start with a linear, anisotropic, and electro-elastic solid for which the coupled constitutive relation can be written as

$$
\begin{gathered}
\sigma_{r}=C_{11} \varepsilon_{r}+C_{12} \varepsilon_{\varphi}-e_{33} E_{r}, \\
\sigma_{\varphi}=C_{12} \varepsilon_{r}+C_{22} \varepsilon_{\varphi}-e_{31} E_{r}, \\
\tau_{r \varphi}=C_{33} \gamma_{r \varphi}-e_{15} E_{\varphi} \\
D_{r}=e_{31} \varepsilon_{\varphi}+e_{33} \varepsilon_{r}+\varepsilon_{33} E_{r}
\end{gathered}
$$




$$
D_{\varphi}=e_{15} \gamma_{r \varphi}+\varepsilon_{11} E_{\varphi},
$$

where $\sigma_{r}, \sigma_{\varphi}$ and $\tau_{r \varphi}$ are the stresses, $\varepsilon_{r}, \varepsilon_{\varphi}$ and $\gamma_{r \varphi}$ are the strains, $D_{r}$ and $D_{\varphi}$ denote the electric displacements, $E_{r}$ and $E_{\varphi}$ are the electric fields, $C_{11}, C_{12}, C_{22}$ and $C_{33}$ are the elastic stiffnesses, $e_{31}, e_{33}$ and $e_{15}$ are the electric permittivity components while $\varepsilon_{11}$ and $\varepsilon_{33}$ stand for the dielectric constants of piezoelectric in the polar coordinate system $(r, \varphi)$.

In the present model the displacement field can be given in the following form

$$
\begin{aligned}
\mathbf{t}(r, \varphi, t)= & w(r, \varphi, t) \mathbf{e}_{r}+u(r, \varphi, t) \mathbf{e}_{\varphi}= \\
= & {\left[v_{0}(\varphi, t)-\frac{r-R}{R}\left(\frac{\partial w_{0}}{\partial \varphi}-v_{0}(\varphi, t)\right)+f(r) v_{1}(\varphi, t)\right] \mathbf{e}_{\varphi}+} \\
& +\left[w_{0}(\varphi, t)+g(r) w_{1}(\varphi, t)\right] \mathbf{e}_{r}
\end{aligned}
$$

where $f(R)=0$ and $g(R)=0$ which means that $\left(v_{0}, w_{0}\right)$ denote the displacement at a point $(R, \varphi)$ on the centre-line along the circumferential and radial directions, respectively.

The terms $f(r) v_{1}(\varphi, t)$ and $g(r) w_{1}(\varphi, t)$ can be thought to be the corrections needed to account for the transverse shear and normal deformation effects, respectively. The functions $f(r)$ and $g(r)$ represent the shape of the corrections through the thickness of the cylinder panel, while $v_{1}(\varphi, t)$ and $w_{1}(\varphi, t)$ determine their distributions along the circumferential direction. The solution of a given problem requires the determination of the unknown functions $v_{0}(\varphi, t), v_{1}(\varphi, t), w_{0}(\varphi, t), w_{1}(\varphi, t), f(r)$ and $g(r)$. By using the standard expressions

$$
\mathbf{t}=w \mathbf{e}_{r}+u \mathbf{e}_{\varphi}, \quad \varepsilon_{\varphi}=\frac{1}{r} \frac{\partial u}{\partial \varphi}+\frac{w}{r}, \quad \gamma_{r \varphi}=\frac{1}{r} \frac{\partial w}{\partial \varphi}+\frac{\partial u}{\partial r}-\frac{u}{r}, \quad \varepsilon_{r}=\frac{\partial w}{\partial r}
$$

and using equation (6) we can determine the components of the strain tensor

$$
\begin{gathered}
\varepsilon_{\varphi}=\frac{1}{r}\left[\frac{\partial v_{0}}{\partial \varphi}-\frac{r-R}{R}\left(\frac{\partial^{2} w_{0}}{\partial \varphi^{2}}-\frac{\partial v_{0}}{\partial \varphi}\right)+f(r) \frac{\partial v_{1}}{\partial \varphi}+w_{0}+g(r) w_{1}(\varphi, t)\right] \\
\gamma_{r \varphi}=\left[\frac{d f}{d r}-\frac{f(r)}{r}\right] v_{1}(\varphi, t)+\frac{g(r)}{r} \frac{\partial w_{1}}{\partial \varphi} \\
\varepsilon_{r}=\frac{d g}{d r} w_{1}(\varphi, t)
\end{gathered}
$$

where $f(r)$ and $g(r)$ are single-valued functions at each point through the thickness.

For a piezoelectric body we have two balance equations to satisfy simultaneously. The balance of momentum and balance of electric charge require the fulfilment of equations

$$
\begin{aligned}
\operatorname{div} \mathbf{T} & =\rho \ddot{\mathbf{t}}, \\
\operatorname{div} \mathbf{D} & =0
\end{aligned}
$$

where $\rho$ is the density, $\mathbf{D}=D_{r}(r, \varphi, t) \mathbf{e}_{r}+D_{\varphi}(r, \varphi, t) \mathbf{e}_{\varphi}$ is the electrical displacement, $\mathbf{T}$ is the stress tensor and the partial derivative with respect to time is denoted by a dot over the letter. 
Assume that the cylinder panel considered is in plane strain. Then the equations of motion 10 and the balance of electric charge (11) can be manipulated into the following forms

$$
\begin{gathered}
\frac{\partial}{\partial r}\left[r^{2} \tau_{r \varphi}\right]+r \frac{\partial \sigma_{\varphi}}{\partial \varphi}=r^{2} \rho \frac{\partial^{2} u}{\partial t^{2}}, \\
r \frac{\partial \sigma_{r}}{\partial r}+\sigma_{r}+\frac{\partial \tau_{r \varphi}}{\partial \varphi}-\sigma_{\varphi}=r \rho \frac{\partial^{2} w}{\partial t^{2}}, \\
\frac{\partial D_{r}}{\partial r}+\frac{D_{r}}{r}+\frac{1}{r} \frac{\partial D_{\varphi}}{\partial \varphi}=0 .
\end{gathered}
$$

The components of the electric field are related to the electrostatic potential $\Phi(r, \varphi, t)$ via the relations

$$
E_{r}=-\frac{\partial \Phi}{\partial r}, \quad E_{\varphi}=-\frac{1}{r} \frac{\partial \Phi}{\partial \varphi}
$$

If the panel is in plain strain $E_{z}=0$.

Here the electric potential can be given the following form

$$
\Phi(r, \varphi, t)=p(r) q(\varphi, t) .
$$

In addition to these equations the boundary conditions on the upper and bottom surface of the panel must be specified. For the study of free vibration these two surfaces are assumed to be traction free. Consequently the following boundary conditions should be prescribed

$$
\begin{gathered}
\tau_{r \varphi}\left(r=R+\frac{h}{2}, \varphi, t\right)=\tau_{r \varphi}\left(r=R-\frac{h}{2}, \varphi, t\right)=0, \\
\sigma_{r}\left(r=R+\frac{h}{2}, \varphi, t\right)=\sigma_{r}\left(r=R-\frac{h}{2}, \varphi, t\right)=0
\end{gathered}
$$

In addition to the mechanical boundary conditions, the electric surface conditions should also be satisfied. This is accomplished in this study by specifying a homogeneous electrostatic potential:

$$
\Phi\left(r=R+\frac{h}{2}, \varphi, t\right)=\Phi\left(r=R-\frac{h}{2}, \varphi, t\right)=0
$$

Substitution of equation 160 into equation $(19)$ yields the following boundary conditions on the upper and bottom surface of the panel

$$
p\left(r=R+\frac{h}{2}\right)=p\left(r=R-\frac{h}{2}\right)=0 .
$$

The Lagrangian for the cylinder panel is given by [6]:

$$
L=\frac{1}{2} \int_{\varphi=0}^{\vartheta} \int_{R-h / 2}^{R+h / 2}\left[-\sigma_{r} \varepsilon_{r}-\sigma_{\varphi} \varepsilon_{\varphi}-\tau_{r \varphi} \gamma_{r \varphi}+D_{\varphi} E_{\varphi}+D_{r} E_{r}+\rho(\dot{\mathbf{t}})^{2}\right] r d r d \varphi .
$$

Substituting equations $(7,9)$ and $(15,16)$ into equations 115 and introducing the results into the Hamilton's principle $\delta \int_{t_{1}}^{t_{2}} L d t=0$ one obtains 


$$
\begin{aligned}
& A_{1,1} \frac{\partial^{4} w_{0}}{\partial \varphi^{4}}+A_{1,2} \frac{\partial^{2} w_{0}}{\partial \varphi^{2}}+A_{1,3} \frac{\partial^{2} w_{1}}{\partial \varphi^{2}}+A_{1,4} \frac{\partial^{3} v_{0}}{\partial \varphi^{3}}+A_{1,5} \frac{\partial^{3} v_{1}}{\partial \varphi^{3}}+ \\
& +A_{1,6} \frac{\partial^{2} q}{\partial \varphi^{2}}+A_{1,7} \frac{\partial v_{0}}{\partial \varphi}+A_{1,8} \frac{\partial v_{1}}{\partial \varphi}+A_{1,9} w_{1}+A_{1,10} w_{0}+A_{1,11} q= \\
& =D_{1,1} \frac{\partial^{4} w_{0}}{\partial \varphi^{2} \partial t^{2}}+D_{1,2} \frac{\partial^{3} v_{0}}{\partial \varphi \partial t^{2}}+D_{1,3} \frac{\partial^{3} v_{1}}{\partial \varphi \partial t^{2}}+D_{1,4} \frac{\partial^{2} w_{0}}{\partial t^{2}}+D_{1,5} \frac{\partial^{2} w_{1}}{\partial t^{2}} \\
& A_{2,1} \frac{\partial^{2} w_{0}}{\partial \varphi^{2}}+A_{2,2} \frac{\partial^{2} w_{1}}{\partial \varphi^{2}}+A_{2,3} \frac{\partial^{2} q}{\partial \varphi^{2}}+A_{2,4} \frac{\partial v_{1}}{\partial \varphi}+A_{2,5} \frac{\partial v_{0}}{\partial \varphi}+ \\
& +A_{2,6} w_{1}+A_{2,7} w_{0}+A_{2,8} q=D_{2,1} \frac{\partial^{2} w_{0}}{\partial t^{2}}+D_{2,2} \frac{\partial^{2} w_{1}}{\partial t^{2}}, \\
& A_{3,1} \frac{\partial^{3} w_{0}}{\partial \varphi^{3}}+A_{3,2} \frac{\partial^{2} v_{1}}{\partial \varphi^{2}}+A_{3,3} \frac{\partial^{2} v_{0}}{\partial \varphi^{2}}+A_{3,4} \frac{\partial w_{0}}{\partial \varphi}+A_{3,5} \frac{\partial w_{1}}{\partial \varphi}+ \\
& +A_{3,6} \frac{\partial q}{\partial \varphi}+A_{3,7} v_{1}=D_{3,1} \frac{\partial^{2} v_{1}}{\partial t^{2}}+D_{3,2} \frac{\partial^{2} v_{0}}{\partial t^{2}}+D_{3,3} \frac{\partial^{3} w_{0}}{\partial \varphi \partial t^{2}}, \\
& A_{4,1} \frac{\partial^{3} w_{0}}{\partial \varphi^{3}}+A_{4,2} \frac{\partial^{2} v_{0}}{\partial \varphi^{2}}+A_{4,3} \frac{\partial^{2} v_{1}}{\partial \varphi^{2}}+A_{4,4} \frac{\partial w_{0}}{\partial \varphi}+ \\
& +A_{4,5} \frac{\partial w_{1}}{\partial \varphi}+A_{4,6} \frac{\partial q}{\partial \varphi}=D_{4,1} \frac{\partial^{3} w_{0}}{\partial \varphi \partial t^{2}}+D_{4,2} \frac{\partial^{2} v_{0}}{\partial t^{2}}+D_{4,3} \frac{\partial^{2} v_{1}}{\partial t^{2}}, \\
& A_{5,1} \frac{\partial^{2} w_{0}}{\partial \varphi^{2}}+A_{5,2} \frac{\partial^{2} w_{1}}{\partial \varphi^{2}}+A_{5,3} \frac{\partial^{2} q}{\partial \varphi^{2}}+A_{5,4} w_{0}+A_{5,5} w_{1}+A_{5,6} q=0,
\end{aligned}
$$

the equations of motion for the four unknown functions, $w_{0}(\varphi, t), w_{1}(\varphi, t), v_{0}(\varphi, t), v_{1}(\varphi, t)$ and $q(\varphi, t)$. In equations 22a 22e $A_{i j}$ and $D_{i j}$ are given in the Appendix.

The section stiffness $K_{1-27}$ and the mass coefficients $M_{1-8}$ are provided by the following equations

$$
\begin{aligned}
K_{[1, \ldots, 10]} & =\int_{R-h / 2}^{R+h / 2} C_{22}\left[\frac{1}{r}, r, \frac{1}{r} f^{2}, \frac{1}{r} g^{2}, 1, \frac{1}{r} f, \frac{1}{r} g, f, g, \frac{1}{r} f g\right] d r, \\
K_{[11]} & =\int_{R-h / 2}^{R+h / 2} C_{11} r\left(\frac{d g}{d r}\right)^{2} d r \\
K_{[12, \ldots, 15]} & =\int_{R-h / 2}^{R+h / 2} C_{12}\left[\frac{d g}{d r}, r \frac{d g}{d r},, f \frac{d g}{d r}, g \frac{d g}{d r}\right] d r, \\
K_{[16, \ldots, 18]} & =\int_{R-h / 2}^{R+h / 2} C_{33}\left[r\left(\frac{d f}{d r}-\frac{f}{r}\right)^{2},\left(\frac{d f}{d r}-\frac{f}{r}\right) g, \frac{1}{r} g^{2}\right] d r \\
K_{[23]} & =\int_{R-h / 2}^{R+h / 2} e_{33} r\left(\frac{d g}{d r}\right)\left(\frac{d p}{d r}\right) d r,
\end{aligned}
$$




$$
\begin{aligned}
K_{[24,25]} & =\int_{R-h / 2}^{R+h / 2} e_{15}\left[p\left(\frac{d f}{d r}-\frac{f}{r}\right), \frac{1}{r} g p\right] d r, \\
K_{[26]} & =\int_{R-h / 2}^{R+h / 2} \varepsilon_{11} \frac{1}{r} p^{2} d r \\
K_{[27]} & =\int_{R-h / 2}^{R+h / 2} \varepsilon_{33} r\left(\frac{d p}{d r}\right)^{2} d r, \\
M_{[1, \ldots, 8]} & =\int_{R-h / 2}^{R+h / 2} \rho\left[r, r^{2}, r^{3}, r f, r^{2} f, r f^{2}, r g, r g^{2}\right] d r,
\end{aligned}
$$

The kinematic and natural boundary conditions specified at $\varphi=0$ and $\varphi=\vartheta$, are given by

$$
\begin{aligned}
v_{0}=0, \quad & F_{11} \frac{\partial^{2} w_{0}}{\partial \varphi^{2}}+F_{12} \frac{\partial v_{0}}{\partial \varphi}+F_{13} \frac{\partial v_{1}}{\partial \varphi}+F_{14} w_{0}+F_{15} w_{1}+F_{16} q=0 \\
w_{0}=0, & F_{21} \frac{\partial^{3} w_{0}}{\partial \varphi^{3}}+F_{22} \frac{\partial^{2} v_{0}}{\partial \varphi^{2}}+F_{23} \frac{\partial^{2} v_{1}}{\partial \varphi^{2}}+F_{24} \frac{\partial^{3} w_{0}}{\partial \varphi^{2} \partial t}+F_{25} \frac{\partial^{2} v_{0}}{\partial t^{2}}+ \\
& +F_{26} \frac{\partial^{2} v_{1}}{\partial t^{2}}+F_{27} \frac{\partial w_{0}}{\partial \varphi}+F_{28} \frac{\partial w_{1}}{\partial \varphi}+F_{29} \frac{\partial q}{\partial \varphi}=0 \\
v_{1}=0, & F_{31} \frac{\partial^{2} w_{0}}{\partial \varphi^{2}}+F_{32} \frac{\partial v_{0}}{\partial \varphi}+F_{33} \frac{\partial v_{1}}{\partial \varphi}+F_{34} w_{0}+F_{35} w_{1}+F_{36} q=0 \\
\frac{\partial w_{0}}{\partial \varphi}=0, & F_{41} \frac{\partial^{2} w_{0}}{\partial \varphi^{2}}+F_{42} \frac{\partial v_{0}}{\partial \varphi}+F_{43} \frac{\partial v_{1}}{\partial \varphi}+F_{44} w_{0}+F_{45} w_{1}+F_{46} q=0 \\
w_{1}=0, & F_{51} \frac{\partial w_{1}}{\partial \varphi}+F_{52} v_{1}+F_{53} \frac{\partial q}{\partial \varphi}=0 \\
q=0, & F_{61} \frac{\partial w_{1}}{\partial \varphi}+F_{62} v_{1}+F_{63} \frac{\partial q}{\partial \varphi}=0
\end{aligned}
$$

where $F_{i j}$ are constants. For the special case of a simply supported curved panel, the first, third and fourth natural boundary conditions are combined with the kinematic condition, $w_{0}=w_{1}=q=0$. In this case the edges of panel are assumed to be grounded.

\section{SOlUtion FOR A SIMPLY SUPPORTED PANEL}

The displacement functions and the potential function satisfying the boundary conditions can be given by

$$
\begin{aligned}
& w_{0}(\varphi, t)=W_{0} \sin \left(k_{n} \varphi\right) e^{i \omega_{n} t} \\
& w_{1}(\varphi, t)=W_{1} \sin \left(k_{n} \varphi\right) e^{i \omega_{n} t} \\
& v_{0}(\varphi, t)=V_{0} \cos \left(k_{n} \varphi\right) e^{i \omega_{n} t} \\
& v_{1}(\varphi, t)=V_{1} \cos \left(k_{n} \varphi\right) e^{i \omega_{n} t}
\end{aligned}
$$




$$
q(\varphi, t)=Q \sin \left(k_{n} \varphi\right) e^{i \omega_{n} t}
$$

where $k_{n}=(n \pi) / \vartheta$. Substitution of equations (25) into equations 22 23) results in a set of five simultaneous, homogeneous algebraic equations with symmetric coefficients. In matrix form, these equations are

$$
\left[-\omega_{n}^{2}[M]+[Y]\right]\{U\}=0, \quad\{U\}=\left\{W_{0}, W_{1}, V_{0}, V_{1}, Q\right\}
$$

where $M_{i j}$ and $Y_{i j}$ are given in the Appendix. While the assumed form of the correction functions $f^{(k)}(r), g^{(k)}(r)$ and $p^{(k)}(r)$ will change from one iteration to the next, in each iteration step they can be treated as known functions. In this phase an estimation of the eigenvalues $W_{0}^{(k)}, W_{1}^{(k)}, V_{0}^{(k)}, V_{1}^{(k)}$ and $Q^{(k)}$ and frequency of vibration $\omega_{n}^{(k)}$ are obtained by solving equation 26. This approximation is then used in the corrector phase in order to improve the predictions for the correction functions $f^{(k+1)}(r), g^{(k+1)}(r)$ and $p^{(k+1)}(r)$.

\section{IMPROVED ESTIMATE FOR CORRECTION FUNCTIONS}

An improved estimate for the correction functions $f^{(k+1)}(r), g^{(k+1)}(r)$ and $p^{(k+1)}(r)$ is derived from the three-dimensional equilibrium equations and the three-dimensional constitutive relations. The transverse shear and normal stress are obtained by integrating the $3 \mathrm{D}$ equilibrium equations $\sqrt{12}, 13)$ in the thickness direction as follows:

$$
\begin{gathered}
\tau_{r \varphi}^{(k+1)}=\frac{1}{r^{2}} \int_{R-h / 2}^{r} r^{2} \rho \frac{\partial^{2} u^{(k)}}{\partial t^{2}}-r \frac{\partial \sigma_{\varphi}^{(k)}}{\partial \varphi} d r+C_{1}, \\
\sigma_{r}^{(k+1)}=\frac{1}{r} \int_{R-h / 2}^{r} r \rho \frac{\partial^{2} w^{(k)}}{\partial t^{2}}+\sigma_{\varphi}^{(k)}-\frac{\partial \tau_{r \varphi}^{(k+1)}}{\partial \varphi} d r+C_{2},
\end{gathered}
$$

where $\sigma_{\varphi}^{(k)}=C_{12} \varepsilon_{r}^{(k)}+C_{22} \varepsilon_{\varphi}^{(k)}-e_{31} E_{r}^{(k)}$ is obtained from equation [2]. The constants $C_{1}$ and $C_{2}$ can be determined from the stress boundary conditions imposed on the outer surfaces of the panel. Owing to equations (17) and (18) we have $C_{1}=C_{2}=0$.

The transverse electric displacement $D_{r}$ is calculated by integrating the $3 \mathrm{D}$ charge equation of electrostatic (14) in the thickness direction

$$
D_{r}^{(k+1)}=\frac{1}{r} \int_{R-h / 2}^{r}-\frac{\partial D_{\varphi}^{(k)}}{\partial \varphi} d r+C_{3},
$$

where $D_{\varphi}^{(k)}=e_{15} \varepsilon_{r \varphi}^{(k)}+\varepsilon_{11} E_{\varphi}^{(k)}$ is obtained from equation (5). The integration constant $C_{3}$ is obtained by setting $D_{r}^{(k+1)}$ at $r=R$ to be equal to the transverse electric displacement of the previous iteration $D_{r}^{(k)}$.

Substitution of equations (7, 9), (15) 16) and (25) in equations (1), (3) and (4) yield the following set of differential equations in terms of the new unknown correction functions $f^{(k+1)}(r), g^{(k+1)}(r)$ and $p^{(k+1)}(r)$.

$$
C_{11} W_{1}^{(k)} \frac{d g^{(k+1)}}{d r}-\frac{C_{12}}{r} k_{n} V_{1}^{(k)} f^{(k+1)}(r)+\frac{C_{12}}{r} W_{1}^{(k)} g^{(k+1)}(r)-e_{33} Q^{(k)} \frac{d p^{(k+1)}}{d r}=
$$




$$
\begin{aligned}
&=\sigma_{r}^{(k+1)}- \frac{C_{12}}{r}\left(-V_{0}^{(k)} k_{n}+\frac{r-R}{R}\left(k_{n}^{2} W_{0}^{(k)}-k_{n} V_{0}^{(k)}\right)+W_{0}^{(k)}\right) \\
& C_{33} V_{1}^{(k)} \frac{d f^{(k+1)}}{d r}-\frac{C_{33}}{r} k_{n} V_{1}^{(k)} f^{(k+1)}(r)+ \\
&+\frac{C_{33}}{r} W_{1}^{(k)} k_{n} g^{(k+1)}(r)+\frac{e_{15}}{r} k_{n} Q^{(k)} p^{(k+1)}=\tau_{r}^{(k+1)} \\
& e_{33} W_{1}^{(k)} \frac{d g^{(k+1)}}{d r}-e_{33} Q^{(k)} \frac{d p^{(k+1)}}{d r}-\frac{e_{31}}{r} k_{n} V_{1}^{(k)} f^{(k+1)}(r)+\frac{e_{31}}{r} W_{1}^{(k)} g^{(k+1)}(r)= \\
&=D_{r}^{(k+1)}-\frac{e_{31}}{r}\left(-V_{0}^{(k)} k_{n}+\frac{r-R}{R}\left(k_{n}^{2} W_{0}^{(k)}-k_{n} V_{0}^{(k)}\right)+W_{0}^{(k)}\right)
\end{aligned}
$$

where $\tau_{r \varphi}^{(k+1)}, \sigma_{r}^{(k+1)}$ and $D_{r}^{(k+1)}$ are obtained from equations 27.29 . As implied above, in addition to these differential equations, the new correction functions must satisfy the following boundary conditions:

$$
f^{(k+1)}(R)=g^{(k+1)}(R)=p^{(k+1)}(R-h / 2)=p^{(k+1)}(R+h / 2)=0 .
$$

The distribution of the new correction functions in the thickness direction is obtained by integrating the equations 30 subject to the boundary conditions of equations (31). The integrals in equations (30) are evaluated numerically using a trapezoidal method. This new estimates of $f^{(k+1)}(r), g^{(k+1)}(r)$ and $p^{(k+1)}(r)$ are used as the correction functions for the next iteration. As with any smeared laminate model,
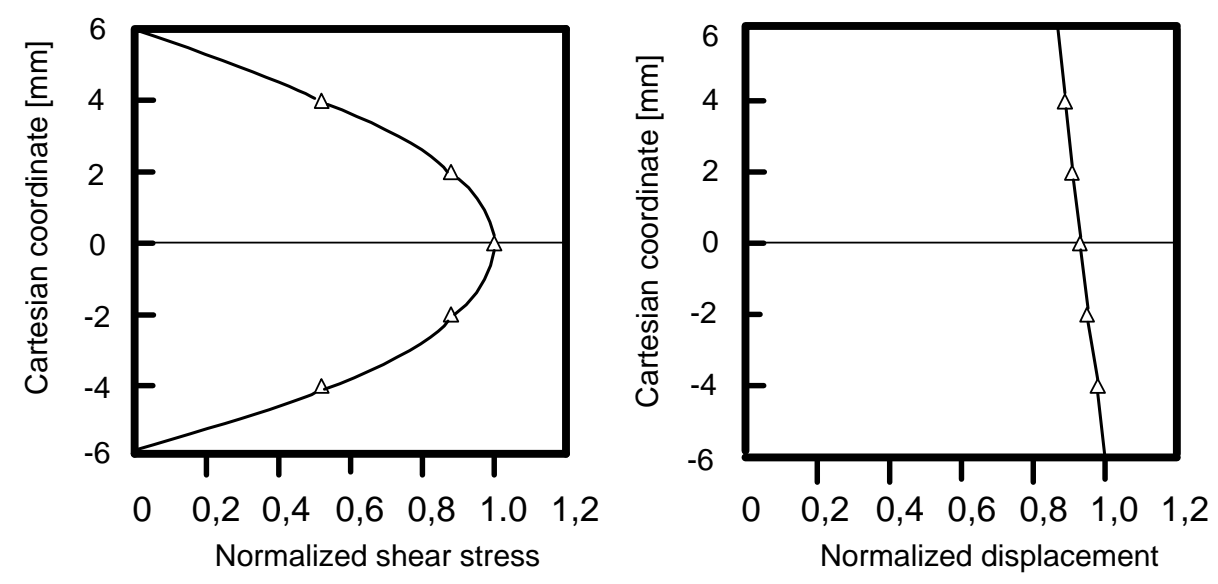

Figure 2. Through-thickness mode shapes for piezoelectric cylinder panel.

there are two distinct ways to calculate the shear stress distribution: from the material constitutive relations; or by elemental stress equilibrium. The ultimate goal of the iterative analysis is the determination of the functions, $f(r)$ and $g(r)$, that causes the two stress distributions to be equal. This defines the convergence point for 
the iterative functions $f(r)$ and $g(r)$, the point at which the stresses and strains are self-consistent.

\section{Results And Discussion}

We consider a layer of piezoelectric material. The input data used here were $h=12.0$ $m m, R=0.8 \mathrm{~m}, L=0.8 \mathrm{~m}, \vartheta=1.0 \mathrm{rad}, C_{11}=6.45 \cdot 10^{10} \mathrm{~N} / \mathrm{m}^{2}, C_{22}=8.13 \cdot 10^{10}$ $\mathrm{N} / \mathrm{m}^{2}, C_{12}=0.0432 \cdot 10^{10} \mathrm{~N} / \mathrm{m}^{2}, C_{33}=2.56 \cdot 10^{10} \mathrm{~N} / \mathrm{m}^{2}, \rho=2.7 \cdot 10^{3} \mathrm{~kg} / \mathrm{m}^{3}$, $e_{33}=15.8 \mathrm{C} / \mathrm{m}^{2}, \quad e_{31}=-5.2 \mathrm{C} / \mathrm{m}^{2}, \quad e_{15}=12.72 \mathrm{C} / \mathrm{m}^{2}, \quad \varepsilon_{11}=1.51 \cdot 10^{-8} \mathrm{~F} / \mathrm{m}$, $\varepsilon_{33}=1.3 \cdot 10^{-8} \mathrm{~F} / \mathrm{m}$. In the example the thickness-to-radius ratio was 0.015 i.e. the panel is considered to be thin and of radius of curvature $R$ of the middle surface, and thickness $h$.

Table 1 Iteration improvement of frequency factor for the panel

\begin{tabular}{|c|c|}
\hline $\begin{array}{c}\text { Iteration } \\
\text { number }\end{array}$ & frequency factor $\omega_{n}^{2}$ \\
\hline 1 & $6.297877 \cdot 10^{4}$ \\
\hline 2 & $6.399191 \cdot 10^{4}$ \\
\hline 3 & $6.386247 \cdot 10^{4}$ \\
\hline 4 & $6.386247 \cdot 10^{4}$ \\
\hline
\end{tabular}

For the minimum vibration frquencies $(n=1)$ representative through-thickness distributions of the elastic and electric field variables are shown.

These have been normalized by dividing through by the maximum value for each field variable. The through-thickness distributions for axial displacement $u$, transverse shear stress $\tau_{r \varphi}$, transverse normal stress $\sigma_{r}$, and transverse electric field $E_{r}$ are shown in Fig. 2-3, respectively, associated with minimum vibration frequencies. The plot for the transverse displacement $w$ is not shown because for the thin panel the distribution is essentially constant throught the thickness.
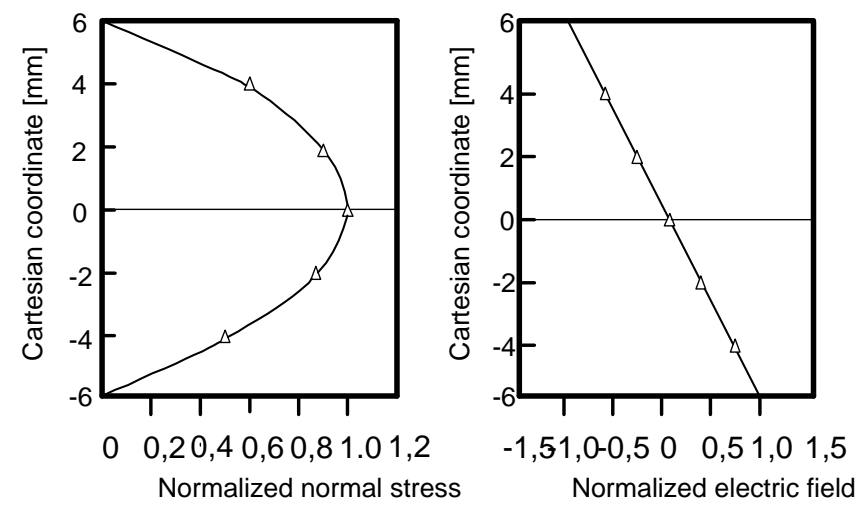

Figure 3. Through-thickness mode shapes for the piezoelectric cylinder panel. 
Since $f(r)$ and $g(r)$ are determined from the previous iteration, the question arises as to what to use for the first iteration. Almost any reasonable displacement assumption is acceptable, however experience has shown that a quadratic shear stress distribution is a very good starting point. Table 1 . shows the improvement in the prediction of the frequency factor $\omega_{n}^{2}$ for the cylinder panel example using a quadratic shear stress as a starting point for the first iteration.

\section{Conclusions}

A new iterative model has been presented for a thin piezoelectric cylindrical panel that can accurately determine the dynamic stress distribution in the panel. This represents an advantage over previous smeared laminate models, in which accurate estimates of the stress field were only possible if the assumed displacement field was a reasonable approximation of the actual displacement field. Accurate determination of the stress and displacement fields is particularly important for "stress critical" calculations such as damping and delamination.

\section{REFERENCES}

1. Cady, W. G.: Piezoelectricity (rev. ed.) Dover, New York, 1964.

2. Nye, N. Y.: Physical Properties of Crystal: Their Representation by tensors and Matrices, Oxford University Press, Oxford, England, 1972.

3. Parton V. Z. and Kundryavtsev, B. A.: Electromagnetoelasticity, Gordon and Breach, New York, 1988.

4. Eer Nisse, E. P.: Resonances of one-nimensional composite piezoelectric and elastic structures. IEEE Transactions on Sonics and Ultrasonics, 14, (1967), 59-67.

5. Holland, R.: Resonant properties of piezoelectric ceramic rectangular parrallelepipeds. Journal of the Acoustical Society of America, 43 (1968), 988-997.

6. Tiersten H. F. Linear Piezoelectric Plate Vibrations. Plenum Press, New York, 1969.

7. Tzou, H. S. and Tseng C. I.: Distributed piezoelectric sensor/actuator design for dynamic measurement/control of distributed parameter systems: A piezoelectric finite element approach. Journal of Sound and Vibration, 138, (1990), 17-34.

8. Tzou, H. S., Tseng C. I. and Wan, G. C.: Distributed structural dynamics control of flexible manipulators-II. Distributed sensor and active electromechanical actuator. Computers and Structures, 35, (1990), 679-687.

9. Robbins D. H. and Reddy, J. N.: Analysis of piezoelectrically actuated beams using a layer-wise displacement theory.1991 Computers and Structures, 41, (1991), 265-279.

10. RedDY, J. N.: A generalization of two-dimensional theories of laminated composite plates. Communication Applied Numerical Mathamatics, 3, (1987), 173-???.

11. Ray, M. C., Rao, K. M. and Samanta, B.: Exact analysis of coupled electroelastic behavior of a piezoelectric plate under cylinder bending. Computers and Structures, $\mathbf{4 5}$, (1992), 667-677.

12. Ray, M. C., Rao, K. M. and Samanta, B.: Exact analysis for static analysis of an intelligent structure under cylinder bending. Computers and Structures, 47, (1993), $1031-1042$. 
13. Sosa, H. A. and Pak, Y. E.: Three dimensional eigenfunction analysis of a crack in a piezoelectric material.International Journal of Solids and Structures, 26, (1990), 1-15.

14. Heylinger, P. and Brooks, S.: Free vibration of piezoelectric laminates in cylinder bending. International Journal of Solids and Structures, 32, (1994), 2945-2960.

15. Heylinger, P., Pei, K. C. and Saravanos, D.: Layerwise mechanics and finite element model for laminated piezoelectric shells. AIAA Journal, 34, (1996), 2353-2360.

16. KovÁcs, P. Z. and PÁczelt, I.: Investigation of the deformation of a multilayered piezoelectric semiring. Journal of Computational and Applied Mechanics, 4, (2002), 159173.

17. Zapfe, J. A. and Lesieutre G. A.: Vibration analyses of laminated beams using an iterative smeared laminate model. Journal of Sound and Vibration, 199, (1997), 275284.

18. Noor, A. K. and Burton, W. S.: Stress and free vibration analyses of multilayered composite plates. Computers and Structures, 11, (1989), 183-204.

19. Lee, K.H. and Cao, L.: 1995 A predictor-corrector zig-zag model for the bending of laminated composite plates.International Journal of Solids and Structures 33, (1995), 879-897.

\section{Appendix A. Constants}

Equations 22 to 23 in the main text are given in terms of $A_{i j}$ and $D_{i j}$ which are presented below:

$$
\begin{aligned}
& A_{1,1}=K_{1}+K_{2}-2 K_{5} / R, \quad A_{1,2}=2 K_{1}-2 K_{5} / R \\
& A_{1,3}=K_{7}+K_{12}-K_{9} / R-K_{13} / R \\
& A_{1,4}=-K_{2}+K_{5} / R, \quad A_{1,5}=K_{6}-K_{8} / R \\
& A_{1,6}=K_{19}-K_{20} / R, \quad A_{1,7}=K_{5} / R \\
& A_{1,8}=K_{6}, \quad A_{1,9}=K_{12}+K_{7}, \quad A_{1,10}=K_{1}, \quad A_{1,11}=K_{19} \\
& A_{2,1}=-K_{7}-K_{12}+K_{9} / R+K_{13} / R, \quad A_{2,2}=K_{18}, \quad A_{2,3}=K_{27}, \\
& A_{2,4}=K_{17}-K_{10}-K_{14}, \quad A_{2,5}=-K_{9} / R-K_{13} / R \\
& A_{2,6}=-K_{4}-K_{11}-K_{15} \\
& A_{2,7}=-K_{7}-K_{12}, \quad A_{2,8}=-K_{22}-K_{23} \\
& A_{3,1}=K_{6}-K_{8} / R, \quad A_{3,2}=K_{3}, \quad A_{3,3}=K_{8} / R \\
& A_{3,4}=K_{6}, \quad A_{3,5}=K_{10}+K_{14}-K_{16} \\
& A_{3,6}=K_{21}-K_{26}, \quad A_{3,7}=-K_{16} \\
& A_{4,1}=K_{5} / R-K_{2}, \quad A_{4,2}=K_{2}, \quad A_{4,3}=K_{8} / R, \quad A_{4,4}=K_{5} / R \\
& A_{4,5}=K_{9} / R+K_{13} / R, A_{4,6}=K_{20} / R \\
& A_{5,1}=K_{20} / R-K_{19}, \quad A_{5,2}=K_{27}, \quad A_{5,3}=-K_{30}, \quad A_{5,4}=-K_{19} \\
& A_{5,5}=-K_{22}-K_{23}, A_{5,6}=K_{31} \\
& D_{1,1}=M_{1}-2 M_{2} / R+M_{3} / R^{2} \\
& D_{1,2}=M_{2} / R-M_{3} / R^{2}, \quad D_{1,3}=M_{4}-M_{5} / R \\
& D_{1,4}=-M_{1}, \quad D_{1,5}=-M_{7}, \quad D_{2,1}=M_{7}, \quad D_{2,2}=M_{8} \\
& D_{3,1}=M_{6}, \quad D_{3,2}=M_{5} / R, \quad D_{3,3}=M_{4}-M_{5} / R \\
& D_{4,1}=M_{2} / R-M_{3} / R^{2}, \quad D_{4,2}=M_{3} / R^{2}, \quad D_{4,3}=M_{5} / R \text {. }
\end{aligned}
$$


Equation 26 in the main text contain the quantities $Y_{i j}$ and $M_{i j}$ which are presented below:

$$
\begin{aligned}
& Y_{11}=k_{n}^{4}\left(K_{1}+K_{2}-2 K_{5} / R\right)-2 k_{n}^{2}\left(K_{1}-K_{5} / R\right)+K_{1} \\
& Y_{12}=Y_{21}=K_{7}+K_{12}-k_{n}^{2}\left(K_{7}+K_{12}-K_{9} / R-K_{13} / R\right) \\
& Y_{13}=Y_{31}=k_{n}^{3}\left(K_{5} / R-K_{2}\right)-k_{n} K_{5} / R \\
& Y_{14}=Y_{41}=k_{n}^{3}\left(K_{6}-K_{8} / R\right)-k_{n} K_{6} \\
& Y_{15}=Y_{51}=K_{19}-k_{n}^{2}\left(K_{19}-K_{20} / R\right) \\
& Y_{22}=k_{n}^{2} K_{18}+K_{4}+K_{11}+K_{15}, \quad Y_{23}=Y_{32}=k_{n}\left(-K_{9} / R-K_{13} / R\right) \\
& Y_{24}=Y_{42}=k_{n}\left(K_{17}-K_{10}-K_{14}\right), \quad Y_{25}=Y_{52}=k_{n}^{2} K_{27}+K_{22}+K_{23} \\
& Y_{33}=k_{n}^{2} K_{2}, \quad Y_{34}=Y_{43}=k_{n}^{2} K_{8} / R, \quad Y_{35}=Y_{53}=-k_{n} K_{20} / R \\
& Y_{44}=k_{n}^{2} K_{3}+K_{16}, \quad Y_{45}=Y_{54}=k_{n}\left(K_{26}-K_{21}\right), \quad Y_{55}=-K_{31}-k_{n}^{2} K_{30} \\
& M_{11}=M_{1}+k_{n}^{2}\left(M_{1}+M_{3} / R^{2}-2 M_{2} / R\right), M_{12}=M_{21}=M_{7} \\
& M_{13}=M_{31}=k_{n}\left(M_{2} / R-M_{3} / R^{2}\right), \quad M_{14}=M_{41}=k_{n}\left(M_{4}-M_{5} / R\right) \\
& M_{33}=M_{3} / R^{2}, \quad M_{34}=M_{43}=M_{5} / R, \quad M_{44}=M_{6}, M_{22}=M_{8} \\
& M_{23}=M_{32}=M_{24}=M_{42}=0 .
\end{aligned}
$$

